\title{
Perception of adults on electronic cigarettes (E-cigarette) in a Malaysian tertiary care centre
}

\begin{abstract}
Background: Tobacco use is a major preventable cause of premature death and disease. The used of electronic cigarettes (e-cigarettes) has gained its popularity recently. Unfortunately, the health impact of e-cigarettes is widely unknown till today. Objective: To explore adult's perceptions of risks and benefits to e-cigarettes. Methods: This is a cross-sectional singlecentre study among young adults in a Malaysian tertiary care centre. A total of 247 young adults in Serdang Hospital were included in this study. A written, self-administered questionnaire was used for data collection. Result: Majority of the respondents of this study consist of Malay female. Although these respondents recorded low usage of both cigarette and e-cigarette, they are highly aware of e-cigarettes. The results showed a high agreement on the perceived risk of e-cigarettes in which majority of them perceived e-cigarettes as harmful to one's health and socially unacceptable. Despite that, a minority of the respondents still believed that e-cigarettes are useful in conforming to the society. Conclusion: This study provides fundamental information on their risk and benefit perception to enable appropriate measures to be carried out in tackling young adults engaging with e-cigarettes.
\end{abstract}

Keyword: Cigarettes; Electronic cigarettes; Perception; Adults 